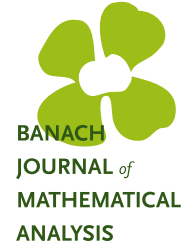

Banach J. Math. Anal. 10 (2016), no. 3, 593-607

http://dx.doi.org/10.1215/17358787-3607420

ISSN: $1735-8787$ (electronic)

http://projecteuclid.org/bjma

ANALYSIS

\title{
SZEGÖ-TYPE DECOMPOSITIONS FOR ISOMETRIES
}

\author{
ZBIGNIEW BURDAK, ${ }^{1}$ MAREK KOSIEK, ${ }^{2}$ \\ PATRYK PAGACZ, $2^{*}$ and MAREK SŁOCIŃSKI ${ }^{2}$ \\ Communicated by F. Kittaneh
}

\begin{abstract}
The notion of Szegö-type properties of positive Borel measures is well known and widely exploited. In this paper, we consider a class of orthogonal decompositions of isometries on Hilbert spaces which correspond to Szegö-type properties of their elementary measures. Our decompositions are closely connected with some special families of invariant subspaces. It is shown that this connection holds for the decomposition constructed in the paper. We illustrate our results with several examples. We also give a short proof of Mlak's theorem on the elementary measures of completely nonunitary contractions.
\end{abstract}

\section{INTRODUCTION AND PRELIMINARIES}

Let $\mathcal{B}(\mathcal{H})$ denote the algebra of all bounded linear operators on a complex Hilbert space $\mathcal{H}$. For a given isometry $V \in \mathcal{B}(\mathcal{H})$, denote by $\mathcal{H}=\mathcal{H}_{u} \oplus \mathcal{H}_{s}$ its Wold decomposition and by $E$ the spectral measure of its minimal unitary extension. For every $x \in \mathcal{H}$ the mapping $\mu_{x}: \mathbb{B}(\mathbb{T}) \ni \omega \mapsto\langle E(\omega) x, x\rangle$ is a positive Borel measure, where $\mathbb{B}(\mathbb{T})$ denotes the $\sigma$-algebra of all Borel subsets of the unit circle $\mathbb{T}$. The measure $\mu_{x}$ is called the elementary measure of $x$ (and $V$ ).

Recall that a unitary operator $U \in \mathcal{B}(\mathcal{K})$ is called a unitary dilation of a contraction $T \in \mathcal{B}(\mathcal{H})$ if

$$
T^{n}=\left.P_{\mathcal{H}} U^{n}\right|_{\mathcal{H}} \quad \text { for } n \in \mathbb{N} \text {, }
$$

Copyright 2016 by the Tusi Mathematical Research Group.

Received Apr. 16, 2015; Accepted Nov. 19, 2015.

${ }^{*}$ Corresponding author.

2010 Mathematics Subject Classification. Primary 47B20; Secondary 47B40, 47A20, 47B37.

Keywords. isometries, Szegö measures, Wold decomposition, wandering vectors, invariant subspaces. 


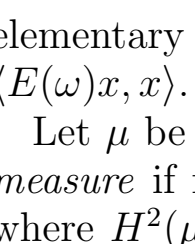

Banach J. Math. Anal. 10 (2016), no. 3, 593-607

http://dx.doi.org/10.1215/17358787-3607420

ISSN: $1735-8787$ (electronic)

http://projecteuclid.org/bjma

ANALYSIS

\title{
SZEGÖ-TYPE DECOMPOSITIONS FOR ISOMETRIES
}

\author{
ZBIGNIEW BURDAK, ${ }^{1}$ MAREK KOSIEK, ${ }^{2}$ \\ PATRYK PAGACZ, $2^{*}$ and MAREK SŁOCIŃSKI ${ }^{2}$ \\ Communicated by F. Kittaneh
}

\begin{abstract}
The notion of Szegö-type properties of positive Borel measures is well known and widely exploited. In this paper, we consider a class of orthogonal decompositions of isometries on Hilbert spaces which correspond to Szegö-type properties of their elementary measures. Our decompositions are closely connected with some special families of invariant subspaces. It is shown that this connection holds for the decomposition constructed in the paper. We illustrate our results with several examples. We also give a short proof of Mlak's theorem on the elementary measures of completely nonunitary contractions.
\end{abstract}

\section{INTRODUCTION AND PRELIMINARIES}

Let $\mathcal{B}(\mathcal{H})$ denote the algebra of all bounded linear operators on a complex Hilbert space $\mathcal{H}$. For a given isometry $V \in \mathcal{B}(\mathcal{H})$, denote by $\mathcal{H}=\mathcal{H}_{u} \oplus \mathcal{H}_{s}$ its Wold decomposition and by $E$ the spectral measure of its minimal unitary extension. For every $x \in \mathcal{H}$ the mapping $\mu_{x}: \mathbb{B}(\mathbb{T}) \ni \omega \mapsto\langle E(\omega) x, x\rangle$ is a positive Borel measure, where $\mathbb{B}(\mathbb{T})$ denotes the $\sigma$-algebra of all Borel subsets of the unit circle $\mathbb{T}$. The measure $\mu_{x}$ is called the elementary measure of $x$ (and $V$ ).

Recall that a unitary operator $U \in \mathcal{B}(\mathcal{K})$ is called a unitary dilation of a contraction $T \in \mathcal{B}(\mathcal{H})$ if

$$
T^{n}=\left.P_{\mathcal{H}} U^{n}\right|_{\mathcal{H}} \quad \text { for } n \in \mathbb{N} \text {, }
$$

Copyright 2016 by the Tusi Mathematical Research Group.

Received Apr. 16, 2015; Accepted Nov. 19, 2015.

${ }^{*}$ Corresponding author.

2010 Mathematics Subject Classification. Primary 47B20; Secondary 47B40, 47A20, 47B37.

Keywords. isometries, Szegö measures, Wold decomposition, wandering vectors, invariant subspaces. 


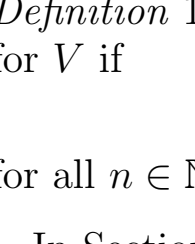

Banach J. Math. Anal. 10 (2016), no. 3, 593-607

http://dx.doi.org/10.1215/17358787-3607420

ISSN: $1735-8787$ (electronic)

http://projecteuclid.org/bjma

ANALYSIS

\title{
SZEGÖ-TYPE DECOMPOSITIONS FOR ISOMETRIES
}

\author{
ZBIGNIEW BURDAK, ${ }^{1}$ MAREK KOSIEK, ${ }^{2}$ \\ PATRYK PAGACZ, $2^{*}$ and MAREK SŁOCIŃSKI ${ }^{2}$ \\ Communicated by F. Kittaneh
}

\begin{abstract}
The notion of Szegö-type properties of positive Borel measures is well known and widely exploited. In this paper, we consider a class of orthogonal decompositions of isometries on Hilbert spaces which correspond to Szegö-type properties of their elementary measures. Our decompositions are closely connected with some special families of invariant subspaces. It is shown that this connection holds for the decomposition constructed in the paper. We illustrate our results with several examples. We also give a short proof of Mlak's theorem on the elementary measures of completely nonunitary contractions.
\end{abstract}

\section{INTRODUCTION AND PRELIMINARIES}

Let $\mathcal{B}(\mathcal{H})$ denote the algebra of all bounded linear operators on a complex Hilbert space $\mathcal{H}$. For a given isometry $V \in \mathcal{B}(\mathcal{H})$, denote by $\mathcal{H}=\mathcal{H}_{u} \oplus \mathcal{H}_{s}$ its Wold decomposition and by $E$ the spectral measure of its minimal unitary extension. For every $x \in \mathcal{H}$ the mapping $\mu_{x}: \mathbb{B}(\mathbb{T}) \ni \omega \mapsto\langle E(\omega) x, x\rangle$ is a positive Borel measure, where $\mathbb{B}(\mathbb{T})$ denotes the $\sigma$-algebra of all Borel subsets of the unit circle $\mathbb{T}$. The measure $\mu_{x}$ is called the elementary measure of $x$ (and $V$ ).

Recall that a unitary operator $U \in \mathcal{B}(\mathcal{K})$ is called a unitary dilation of a contraction $T \in \mathcal{B}(\mathcal{H})$ if

$$
T^{n}=\left.P_{\mathcal{H}} U^{n}\right|_{\mathcal{H}} \quad \text { for } n \in \mathbb{N} \text {, }
$$

Copyright 2016 by the Tusi Mathematical Research Group.

Received Apr. 16, 2015; Accepted Nov. 19, 2015.

${ }^{*}$ Corresponding author.

2010 Mathematics Subject Classification. Primary 47B20; Secondary 47B40, 47A20, 47B37.

Keywords. isometries, Szegö measures, Wold decomposition, wandering vectors, invariant subspaces. 


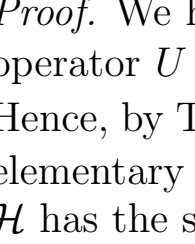

Banach J. Math. Anal. 10 (2016), no. 3, 593-607

http://dx.doi.org/10.1215/17358787-3607420

ISSN: $1735-8787$ (electronic)

http://projecteuclid.org/bjma

ANALYSIS

\title{
SZEGÖ-TYPE DECOMPOSITIONS FOR ISOMETRIES
}

\author{
ZBIGNIEW BURDAK, ${ }^{1}$ MAREK KOSIEK, ${ }^{2}$ \\ PATRYK PAGACZ, $2^{*}$ and MAREK SŁOCIŃSKI ${ }^{2}$ \\ Communicated by F. Kittaneh
}

\begin{abstract}
The notion of Szegö-type properties of positive Borel measures is well known and widely exploited. In this paper, we consider a class of orthogonal decompositions of isometries on Hilbert spaces which correspond to Szegö-type properties of their elementary measures. Our decompositions are closely connected with some special families of invariant subspaces. It is shown that this connection holds for the decomposition constructed in the paper. We illustrate our results with several examples. We also give a short proof of Mlak's theorem on the elementary measures of completely nonunitary contractions.
\end{abstract}

\section{INTRODUCTION AND PRELIMINARIES}

Let $\mathcal{B}(\mathcal{H})$ denote the algebra of all bounded linear operators on a complex Hilbert space $\mathcal{H}$. For a given isometry $V \in \mathcal{B}(\mathcal{H})$, denote by $\mathcal{H}=\mathcal{H}_{u} \oplus \mathcal{H}_{s}$ its Wold decomposition and by $E$ the spectral measure of its minimal unitary extension. For every $x \in \mathcal{H}$ the mapping $\mu_{x}: \mathbb{B}(\mathbb{T}) \ni \omega \mapsto\langle E(\omega) x, x\rangle$ is a positive Borel measure, where $\mathbb{B}(\mathbb{T})$ denotes the $\sigma$-algebra of all Borel subsets of the unit circle $\mathbb{T}$. The measure $\mu_{x}$ is called the elementary measure of $x$ (and $V$ ).

Recall that a unitary operator $U \in \mathcal{B}(\mathcal{K})$ is called a unitary dilation of a contraction $T \in \mathcal{B}(\mathcal{H})$ if

$$
T^{n}=\left.P_{\mathcal{H}} U^{n}\right|_{\mathcal{H}} \quad \text { for } n \in \mathbb{N} \text {, }
$$

Copyright 2016 by the Tusi Mathematical Research Group.

Received Apr. 16, 2015; Accepted Nov. 19, 2015.

${ }^{*}$ Corresponding author.

2010 Mathematics Subject Classification. Primary 47B20; Secondary 47B40, 47A20, 47B37.

Keywords. isometries, Szegö measures, Wold decomposition, wandering vectors, invariant subspaces. 


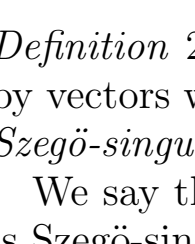

Banach J. Math. Anal. 10 (2016), no. 3, 593-607

http://dx.doi.org/10.1215/17358787-3607420

ISSN: $1735-8787$ (electronic)

http://projecteuclid.org/bjma

ANALYSIS

\title{
SZEGÖ-TYPE DECOMPOSITIONS FOR ISOMETRIES
}

\author{
ZBIGNIEW BURDAK, ${ }^{1}$ MAREK KOSIEK, ${ }^{2}$ \\ PATRYK PAGACZ, $2^{*}$ and MAREK SŁOCIŃSKI ${ }^{2}$ \\ Communicated by F. Kittaneh
}

\begin{abstract}
The notion of Szegö-type properties of positive Borel measures is well known and widely exploited. In this paper, we consider a class of orthogonal decompositions of isometries on Hilbert spaces which correspond to Szegö-type properties of their elementary measures. Our decompositions are closely connected with some special families of invariant subspaces. It is shown that this connection holds for the decomposition constructed in the paper. We illustrate our results with several examples. We also give a short proof of Mlak's theorem on the elementary measures of completely nonunitary contractions.
\end{abstract}

\section{INTRODUCTION AND PRELIMINARIES}

Let $\mathcal{B}(\mathcal{H})$ denote the algebra of all bounded linear operators on a complex Hilbert space $\mathcal{H}$. For a given isometry $V \in \mathcal{B}(\mathcal{H})$, denote by $\mathcal{H}=\mathcal{H}_{u} \oplus \mathcal{H}_{s}$ its Wold decomposition and by $E$ the spectral measure of its minimal unitary extension. For every $x \in \mathcal{H}$ the mapping $\mu_{x}: \mathbb{B}(\mathbb{T}) \ni \omega \mapsto\langle E(\omega) x, x\rangle$ is a positive Borel measure, where $\mathbb{B}(\mathbb{T})$ denotes the $\sigma$-algebra of all Borel subsets of the unit circle $\mathbb{T}$. The measure $\mu_{x}$ is called the elementary measure of $x$ (and $V$ ).

Recall that a unitary operator $U \in \mathcal{B}(\mathcal{K})$ is called a unitary dilation of a contraction $T \in \mathcal{B}(\mathcal{H})$ if

$$
T^{n}=\left.P_{\mathcal{H}} U^{n}\right|_{\mathcal{H}} \quad \text { for } n \in \mathbb{N} \text {, }
$$

Copyright 2016 by the Tusi Mathematical Research Group.

Received Apr. 16, 2015; Accepted Nov. 19, 2015.

${ }^{*}$ Corresponding author.

2010 Mathematics Subject Classification. Primary 47B20; Secondary 47B40, 47A20, 47B37.

Keywords. isometries, Szegö measures, Wold decomposition, wandering vectors, invariant subspaces. 


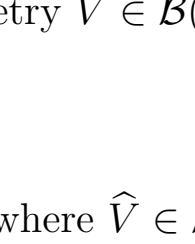

Banach J. Math. Anal. 10 (2016), no. 3, 593-607

http://dx.doi.org/10.1215/17358787-3607420

ISSN: $1735-8787$ (electronic)

http://projecteuclid.org/bjma

ANALYSIS

\title{
SZEGÖ-TYPE DECOMPOSITIONS FOR ISOMETRIES
}

\author{
ZBIGNIEW BURDAK, ${ }^{1}$ MAREK KOSIEK, ${ }^{2}$ \\ PATRYK PAGACZ, $2^{*}$ and MAREK SŁOCIŃSKI ${ }^{2}$ \\ Communicated by F. Kittaneh
}

\begin{abstract}
The notion of Szegö-type properties of positive Borel measures is well known and widely exploited. In this paper, we consider a class of orthogonal decompositions of isometries on Hilbert spaces which correspond to Szegö-type properties of their elementary measures. Our decompositions are closely connected with some special families of invariant subspaces. It is shown that this connection holds for the decomposition constructed in the paper. We illustrate our results with several examples. We also give a short proof of Mlak's theorem on the elementary measures of completely nonunitary contractions.
\end{abstract}

\section{INTRODUCTION AND PRELIMINARIES}

Let $\mathcal{B}(\mathcal{H})$ denote the algebra of all bounded linear operators on a complex Hilbert space $\mathcal{H}$. For a given isometry $V \in \mathcal{B}(\mathcal{H})$, denote by $\mathcal{H}=\mathcal{H}_{u} \oplus \mathcal{H}_{s}$ its Wold decomposition and by $E$ the spectral measure of its minimal unitary extension. For every $x \in \mathcal{H}$ the mapping $\mu_{x}: \mathbb{B}(\mathbb{T}) \ni \omega \mapsto\langle E(\omega) x, x\rangle$ is a positive Borel measure, where $\mathbb{B}(\mathbb{T})$ denotes the $\sigma$-algebra of all Borel subsets of the unit circle $\mathbb{T}$. The measure $\mu_{x}$ is called the elementary measure of $x$ (and $V$ ).

Recall that a unitary operator $U \in \mathcal{B}(\mathcal{K})$ is called a unitary dilation of a contraction $T \in \mathcal{B}(\mathcal{H})$ if

$$
T^{n}=\left.P_{\mathcal{H}} U^{n}\right|_{\mathcal{H}} \quad \text { for } n \in \mathbb{N} \text {, }
$$

Copyright 2016 by the Tusi Mathematical Research Group.

Received Apr. 16, 2015; Accepted Nov. 19, 2015.

${ }^{*}$ Corresponding author.

2010 Mathematics Subject Classification. Primary 47B20; Secondary 47B40, 47A20, 47B37.

Keywords. isometries, Szegö measures, Wold decomposition, wandering vectors, invariant subspaces. 


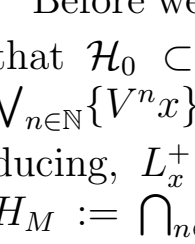

Banach J. Math. Anal. 10 (2016), no. 3, 593-607

http://dx.doi.org/10.1215/17358787-3607420

ISSN: $1735-8787$ (electronic)

http://projecteuclid.org/bjma

ANALYSIS

\title{
SZEGÖ-TYPE DECOMPOSITIONS FOR ISOMETRIES
}

\author{
ZBIGNIEW BURDAK, ${ }^{1}$ MAREK KOSIEK, ${ }^{2}$ \\ PATRYK PAGACZ, $2^{*}$ and MAREK SŁOCIŃSKI ${ }^{2}$ \\ Communicated by F. Kittaneh
}

\begin{abstract}
The notion of Szegö-type properties of positive Borel measures is well known and widely exploited. In this paper, we consider a class of orthogonal decompositions of isometries on Hilbert spaces which correspond to Szegö-type properties of their elementary measures. Our decompositions are closely connected with some special families of invariant subspaces. It is shown that this connection holds for the decomposition constructed in the paper. We illustrate our results with several examples. We also give a short proof of Mlak's theorem on the elementary measures of completely nonunitary contractions.
\end{abstract}

\section{INTRODUCTION AND PRELIMINARIES}

Let $\mathcal{B}(\mathcal{H})$ denote the algebra of all bounded linear operators on a complex Hilbert space $\mathcal{H}$. For a given isometry $V \in \mathcal{B}(\mathcal{H})$, denote by $\mathcal{H}=\mathcal{H}_{u} \oplus \mathcal{H}_{s}$ its Wold decomposition and by $E$ the spectral measure of its minimal unitary extension. For every $x \in \mathcal{H}$ the mapping $\mu_{x}: \mathbb{B}(\mathbb{T}) \ni \omega \mapsto\langle E(\omega) x, x\rangle$ is a positive Borel measure, where $\mathbb{B}(\mathbb{T})$ denotes the $\sigma$-algebra of all Borel subsets of the unit circle $\mathbb{T}$. The measure $\mu_{x}$ is called the elementary measure of $x$ (and $V$ ).

Recall that a unitary operator $U \in \mathcal{B}(\mathcal{K})$ is called a unitary dilation of a contraction $T \in \mathcal{B}(\mathcal{H})$ if

$$
T^{n}=\left.P_{\mathcal{H}} U^{n}\right|_{\mathcal{H}} \quad \text { for } n \in \mathbb{N} \text {, }
$$

Copyright 2016 by the Tusi Mathematical Research Group.

Received Apr. 16, 2015; Accepted Nov. 19, 2015.

${ }^{*}$ Corresponding author.

2010 Mathematics Subject Classification. Primary 47B20; Secondary 47B40, 47A20, 47B37.

Keywords. isometries, Szegö measures, Wold decomposition, wandering vectors, invariant subspaces. 


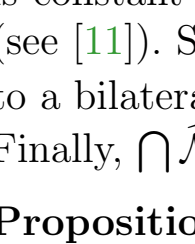

Banach J. Math. Anal. 10 (2016), no. 3, 593-607

http://dx.doi.org/10.1215/17358787-3607420

ISSN: $1735-8787$ (electronic)

http://projecteuclid.org/bjma

ANALYSIS

\title{
SZEGÖ-TYPE DECOMPOSITIONS FOR ISOMETRIES
}

\author{
ZBIGNIEW BURDAK, ${ }^{1}$ MAREK KOSIEK, ${ }^{2}$ \\ PATRYK PAGACZ, $2^{*}$ and MAREK SŁOCIŃSKI ${ }^{2}$ \\ Communicated by F. Kittaneh
}

\begin{abstract}
The notion of Szegö-type properties of positive Borel measures is well known and widely exploited. In this paper, we consider a class of orthogonal decompositions of isometries on Hilbert spaces which correspond to Szegö-type properties of their elementary measures. Our decompositions are closely connected with some special families of invariant subspaces. It is shown that this connection holds for the decomposition constructed in the paper. We illustrate our results with several examples. We also give a short proof of Mlak's theorem on the elementary measures of completely nonunitary contractions.
\end{abstract}

\section{INTRODUCTION AND PRELIMINARIES}

Let $\mathcal{B}(\mathcal{H})$ denote the algebra of all bounded linear operators on a complex Hilbert space $\mathcal{H}$. For a given isometry $V \in \mathcal{B}(\mathcal{H})$, denote by $\mathcal{H}=\mathcal{H}_{u} \oplus \mathcal{H}_{s}$ its Wold decomposition and by $E$ the spectral measure of its minimal unitary extension. For every $x \in \mathcal{H}$ the mapping $\mu_{x}: \mathbb{B}(\mathbb{T}) \ni \omega \mapsto\langle E(\omega) x, x\rangle$ is a positive Borel measure, where $\mathbb{B}(\mathbb{T})$ denotes the $\sigma$-algebra of all Borel subsets of the unit circle $\mathbb{T}$. The measure $\mu_{x}$ is called the elementary measure of $x$ (and $V$ ).

Recall that a unitary operator $U \in \mathcal{B}(\mathcal{K})$ is called a unitary dilation of a contraction $T \in \mathcal{B}(\mathcal{H})$ if

$$
T^{n}=\left.P_{\mathcal{H}} U^{n}\right|_{\mathcal{H}} \quad \text { for } n \in \mathbb{N} \text {, }
$$

Copyright 2016 by the Tusi Mathematical Research Group.

Received Apr. 16, 2015; Accepted Nov. 19, 2015.

${ }^{*}$ Corresponding author.

2010 Mathematics Subject Classification. Primary 47B20; Secondary 47B40, 47A20, 47B37.

Keywords. isometries, Szegö measures, Wold decomposition, wandering vectors, invariant subspaces. 


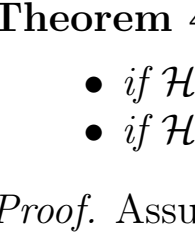

Banach J. Math. Anal. 10 (2016), no. 3, 593-607

http://dx.doi.org/10.1215/17358787-3607420

ISSN: $1735-8787$ (electronic)

http://projecteuclid.org/bjma

ANALYSIS

\title{
SZEGÖ-TYPE DECOMPOSITIONS FOR ISOMETRIES
}

\author{
ZBIGNIEW BURDAK, ${ }^{1}$ MAREK KOSIEK, ${ }^{2}$ \\ PATRYK PAGACZ, $2^{*}$ and MAREK SŁOCIŃSKI ${ }^{2}$ \\ Communicated by F. Kittaneh
}

\begin{abstract}
The notion of Szegö-type properties of positive Borel measures is well known and widely exploited. In this paper, we consider a class of orthogonal decompositions of isometries on Hilbert spaces which correspond to Szegö-type properties of their elementary measures. Our decompositions are closely connected with some special families of invariant subspaces. It is shown that this connection holds for the decomposition constructed in the paper. We illustrate our results with several examples. We also give a short proof of Mlak's theorem on the elementary measures of completely nonunitary contractions.
\end{abstract}

\section{INTRODUCTION AND PRELIMINARIES}

Let $\mathcal{B}(\mathcal{H})$ denote the algebra of all bounded linear operators on a complex Hilbert space $\mathcal{H}$. For a given isometry $V \in \mathcal{B}(\mathcal{H})$, denote by $\mathcal{H}=\mathcal{H}_{u} \oplus \mathcal{H}_{s}$ its Wold decomposition and by $E$ the spectral measure of its minimal unitary extension. For every $x \in \mathcal{H}$ the mapping $\mu_{x}: \mathbb{B}(\mathbb{T}) \ni \omega \mapsto\langle E(\omega) x, x\rangle$ is a positive Borel measure, where $\mathbb{B}(\mathbb{T})$ denotes the $\sigma$-algebra of all Borel subsets of the unit circle $\mathbb{T}$. The measure $\mu_{x}$ is called the elementary measure of $x$ (and $V$ ).

Recall that a unitary operator $U \in \mathcal{B}(\mathcal{K})$ is called a unitary dilation of a contraction $T \in \mathcal{B}(\mathcal{H})$ if

$$
T^{n}=\left.P_{\mathcal{H}} U^{n}\right|_{\mathcal{H}} \quad \text { for } n \in \mathbb{N} \text {, }
$$

Copyright 2016 by the Tusi Mathematical Research Group.

Received Apr. 16, 2015; Accepted Nov. 19, 2015.

${ }^{*}$ Corresponding author.

2010 Mathematics Subject Classification. Primary 47B20; Secondary 47B40, 47A20, 47B37.

Keywords. isometries, Szegö measures, Wold decomposition, wandering vectors, invariant subspaces. 


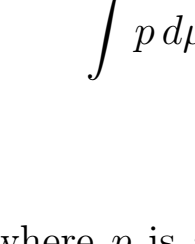

Banach J. Math. Anal. 10 (2016), no. 3, 593-607

http://dx.doi.org/10.1215/17358787-3607420

ISSN: $1735-8787$ (electronic)

http://projecteuclid.org/bjma

ANALYSIS

\title{
SZEGÖ-TYPE DECOMPOSITIONS FOR ISOMETRIES
}

\author{
ZBIGNIEW BURDAK, ${ }^{1}$ MAREK KOSIEK, ${ }^{2}$ \\ PATRYK PAGACZ, $2^{*}$ and MAREK SŁOCIŃSKI ${ }^{2}$ \\ Communicated by F. Kittaneh
}

\begin{abstract}
The notion of Szegö-type properties of positive Borel measures is well known and widely exploited. In this paper, we consider a class of orthogonal decompositions of isometries on Hilbert spaces which correspond to Szegö-type properties of their elementary measures. Our decompositions are closely connected with some special families of invariant subspaces. It is shown that this connection holds for the decomposition constructed in the paper. We illustrate our results with several examples. We also give a short proof of Mlak's theorem on the elementary measures of completely nonunitary contractions.
\end{abstract}

\section{INTRODUCTION AND PRELIMINARIES}

Let $\mathcal{B}(\mathcal{H})$ denote the algebra of all bounded linear operators on a complex Hilbert space $\mathcal{H}$. For a given isometry $V \in \mathcal{B}(\mathcal{H})$, denote by $\mathcal{H}=\mathcal{H}_{u} \oplus \mathcal{H}_{s}$ its Wold decomposition and by $E$ the spectral measure of its minimal unitary extension. For every $x \in \mathcal{H}$ the mapping $\mu_{x}: \mathbb{B}(\mathbb{T}) \ni \omega \mapsto\langle E(\omega) x, x\rangle$ is a positive Borel measure, where $\mathbb{B}(\mathbb{T})$ denotes the $\sigma$-algebra of all Borel subsets of the unit circle $\mathbb{T}$. The measure $\mu_{x}$ is called the elementary measure of $x$ (and $V$ ).

Recall that a unitary operator $U \in \mathcal{B}(\mathcal{K})$ is called a unitary dilation of a contraction $T \in \mathcal{B}(\mathcal{H})$ if

$$
T^{n}=\left.P_{\mathcal{H}} U^{n}\right|_{\mathcal{H}} \quad \text { for } n \in \mathbb{N} \text {, }
$$

Copyright 2016 by the Tusi Mathematical Research Group.

Received Apr. 16, 2015; Accepted Nov. 19, 2015.

${ }^{*}$ Corresponding author.

2010 Mathematics Subject Classification. Primary 47B20; Secondary 47B40, 47A20, 47B37.

Keywords. isometries, Szegö measures, Wold decomposition, wandering vectors, invariant subspaces. 


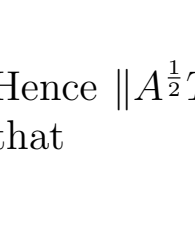

Banach J. Math. Anal. 10 (2016), no. 3, 593-607

http://dx.doi.org/10.1215/17358787-3607420

ISSN: $1735-8787$ (electronic)

http://projecteuclid.org/bjma

ANALYSIS

\title{
SZEGÖ-TYPE DECOMPOSITIONS FOR ISOMETRIES
}

\author{
ZBIGNIEW BURDAK, ${ }^{1}$ MAREK KOSIEK, ${ }^{2}$ \\ PATRYK PAGACZ, $2^{*}$ and MAREK SŁOCIŃSKI ${ }^{2}$ \\ Communicated by F. Kittaneh
}

\begin{abstract}
The notion of Szegö-type properties of positive Borel measures is well known and widely exploited. In this paper, we consider a class of orthogonal decompositions of isometries on Hilbert spaces which correspond to Szegö-type properties of their elementary measures. Our decompositions are closely connected with some special families of invariant subspaces. It is shown that this connection holds for the decomposition constructed in the paper. We illustrate our results with several examples. We also give a short proof of Mlak's theorem on the elementary measures of completely nonunitary contractions.
\end{abstract}

\section{INTRODUCTION AND PRELIMINARIES}

Let $\mathcal{B}(\mathcal{H})$ denote the algebra of all bounded linear operators on a complex Hilbert space $\mathcal{H}$. For a given isometry $V \in \mathcal{B}(\mathcal{H})$, denote by $\mathcal{H}=\mathcal{H}_{u} \oplus \mathcal{H}_{s}$ its Wold decomposition and by $E$ the spectral measure of its minimal unitary extension. For every $x \in \mathcal{H}$ the mapping $\mu_{x}: \mathbb{B}(\mathbb{T}) \ni \omega \mapsto\langle E(\omega) x, x\rangle$ is a positive Borel measure, where $\mathbb{B}(\mathbb{T})$ denotes the $\sigma$-algebra of all Borel subsets of the unit circle $\mathbb{T}$. The measure $\mu_{x}$ is called the elementary measure of $x$ (and $V$ ).

Recall that a unitary operator $U \in \mathcal{B}(\mathcal{K})$ is called a unitary dilation of a contraction $T \in \mathcal{B}(\mathcal{H})$ if

$$
T^{n}=\left.P_{\mathcal{H}} U^{n}\right|_{\mathcal{H}} \quad \text { for } n \in \mathbb{N} \text {, }
$$

Copyright 2016 by the Tusi Mathematical Research Group.

Received Apr. 16, 2015; Accepted Nov. 19, 2015.

${ }^{*}$ Corresponding author.

2010 Mathematics Subject Classification. Primary 47B20; Secondary 47B40, 47A20, 47B37.

Keywords. isometries, Szegö measures, Wold decomposition, wandering vectors, invariant subspaces. 


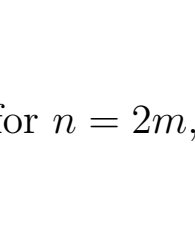

Banach J. Math. Anal. 10 (2016), no. 3, 593-607

http://dx.doi.org/10.1215/17358787-3607420

ISSN: $1735-8787$ (electronic)

http://projecteuclid.org/bjma

ANALYSIS

\title{
SZEGÖ-TYPE DECOMPOSITIONS FOR ISOMETRIES
}

\author{
ZBIGNIEW BURDAK, ${ }^{1}$ MAREK KOSIEK, ${ }^{2}$ \\ PATRYK PAGACZ, $2^{*}$ and MAREK SŁOCIŃSKI ${ }^{2}$ \\ Communicated by F. Kittaneh
}

\begin{abstract}
The notion of Szegö-type properties of positive Borel measures is well known and widely exploited. In this paper, we consider a class of orthogonal decompositions of isometries on Hilbert spaces which correspond to Szegö-type properties of their elementary measures. Our decompositions are closely connected with some special families of invariant subspaces. It is shown that this connection holds for the decomposition constructed in the paper. We illustrate our results with several examples. We also give a short proof of Mlak's theorem on the elementary measures of completely nonunitary contractions.
\end{abstract}

\section{INTRODUCTION AND PRELIMINARIES}

Let $\mathcal{B}(\mathcal{H})$ denote the algebra of all bounded linear operators on a complex Hilbert space $\mathcal{H}$. For a given isometry $V \in \mathcal{B}(\mathcal{H})$, denote by $\mathcal{H}=\mathcal{H}_{u} \oplus \mathcal{H}_{s}$ its Wold decomposition and by $E$ the spectral measure of its minimal unitary extension. For every $x \in \mathcal{H}$ the mapping $\mu_{x}: \mathbb{B}(\mathbb{T}) \ni \omega \mapsto\langle E(\omega) x, x\rangle$ is a positive Borel measure, where $\mathbb{B}(\mathbb{T})$ denotes the $\sigma$-algebra of all Borel subsets of the unit circle $\mathbb{T}$. The measure $\mu_{x}$ is called the elementary measure of $x$ (and $V$ ).

Recall that a unitary operator $U \in \mathcal{B}(\mathcal{K})$ is called a unitary dilation of a contraction $T \in \mathcal{B}(\mathcal{H})$ if

$$
T^{n}=\left.P_{\mathcal{H}} U^{n}\right|_{\mathcal{H}} \quad \text { for } n \in \mathbb{N} \text {, }
$$

Copyright 2016 by the Tusi Mathematical Research Group.

Received Apr. 16, 2015; Accepted Nov. 19, 2015.

${ }^{*}$ Corresponding author.

2010 Mathematics Subject Classification. Primary 47B20; Secondary 47B40, 47A20, 47B37.

Keywords. isometries, Szegö measures, Wold decomposition, wandering vectors, invariant subspaces. 


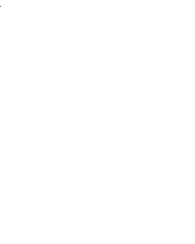

Banach J. Math. Anal. 10 (2016), no. 3, 593-607

http://dx.doi.org/10.1215/17358787-3607420

ISSN: $1735-8787$ (electronic)

http://projecteuclid.org/bjma

ANALYSIS

\title{
SZEGÖ-TYPE DECOMPOSITIONS FOR ISOMETRIES
}

\author{
ZBIGNIEW BURDAK, ${ }^{1}$ MAREK KOSIEK, ${ }^{2}$ \\ PATRYK PAGACZ, $2^{*}$ and MAREK SŁOCIŃSKI ${ }^{2}$ \\ Communicated by F. Kittaneh
}

\begin{abstract}
The notion of Szegö-type properties of positive Borel measures is well known and widely exploited. In this paper, we consider a class of orthogonal decompositions of isometries on Hilbert spaces which correspond to Szegö-type properties of their elementary measures. Our decompositions are closely connected with some special families of invariant subspaces. It is shown that this connection holds for the decomposition constructed in the paper. We illustrate our results with several examples. We also give a short proof of Mlak's theorem on the elementary measures of completely nonunitary contractions.
\end{abstract}

\section{INTRODUCTION AND PRELIMINARIES}

Let $\mathcal{B}(\mathcal{H})$ denote the algebra of all bounded linear operators on a complex Hilbert space $\mathcal{H}$. For a given isometry $V \in \mathcal{B}(\mathcal{H})$, denote by $\mathcal{H}=\mathcal{H}_{u} \oplus \mathcal{H}_{s}$ its Wold decomposition and by $E$ the spectral measure of its minimal unitary extension. For every $x \in \mathcal{H}$ the mapping $\mu_{x}: \mathbb{B}(\mathbb{T}) \ni \omega \mapsto\langle E(\omega) x, x\rangle$ is a positive Borel measure, where $\mathbb{B}(\mathbb{T})$ denotes the $\sigma$-algebra of all Borel subsets of the unit circle $\mathbb{T}$. The measure $\mu_{x}$ is called the elementary measure of $x$ (and $V$ ).

Recall that a unitary operator $U \in \mathcal{B}(\mathcal{K})$ is called a unitary dilation of a contraction $T \in \mathcal{B}(\mathcal{H})$ if

$$
T^{n}=\left.P_{\mathcal{H}} U^{n}\right|_{\mathcal{H}} \quad \text { for } n \in \mathbb{N} \text {, }
$$

Copyright 2016 by the Tusi Mathematical Research Group.

Received Apr. 16, 2015; Accepted Nov. 19, 2015.

${ }^{*}$ Corresponding author.

2010 Mathematics Subject Classification. Primary 47B20; Secondary 47B40, 47A20, 47B37.

Keywords. isometries, Szegö measures, Wold decomposition, wandering vectors, invariant subspaces. 


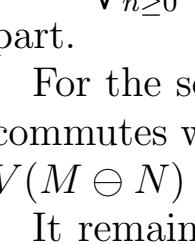

Banach J. Math. Anal. 10 (2016), no. 3, 593-607

http://dx.doi.org/10.1215/17358787-3607420

ISSN: $1735-8787$ (electronic)

http://projecteuclid.org/bjma

ANALYSIS

\title{
SZEGÖ-TYPE DECOMPOSITIONS FOR ISOMETRIES
}

\author{
ZBIGNIEW BURDAK, ${ }^{1}$ MAREK KOSIEK, ${ }^{2}$ \\ PATRYK PAGACZ, $2^{*}$ and MAREK SŁOCIŃSKI ${ }^{2}$ \\ Communicated by F. Kittaneh
}

\begin{abstract}
The notion of Szegö-type properties of positive Borel measures is well known and widely exploited. In this paper, we consider a class of orthogonal decompositions of isometries on Hilbert spaces which correspond to Szegö-type properties of their elementary measures. Our decompositions are closely connected with some special families of invariant subspaces. It is shown that this connection holds for the decomposition constructed in the paper. We illustrate our results with several examples. We also give a short proof of Mlak's theorem on the elementary measures of completely nonunitary contractions.
\end{abstract}

\section{INTRODUCTION AND PRELIMINARIES}

Let $\mathcal{B}(\mathcal{H})$ denote the algebra of all bounded linear operators on a complex Hilbert space $\mathcal{H}$. For a given isometry $V \in \mathcal{B}(\mathcal{H})$, denote by $\mathcal{H}=\mathcal{H}_{u} \oplus \mathcal{H}_{s}$ its Wold decomposition and by $E$ the spectral measure of its minimal unitary extension. For every $x \in \mathcal{H}$ the mapping $\mu_{x}: \mathbb{B}(\mathbb{T}) \ni \omega \mapsto\langle E(\omega) x, x\rangle$ is a positive Borel measure, where $\mathbb{B}(\mathbb{T})$ denotes the $\sigma$-algebra of all Borel subsets of the unit circle $\mathbb{T}$. The measure $\mu_{x}$ is called the elementary measure of $x$ (and $V$ ).

Recall that a unitary operator $U \in \mathcal{B}(\mathcal{K})$ is called a unitary dilation of a contraction $T \in \mathcal{B}(\mathcal{H})$ if

$$
T^{n}=\left.P_{\mathcal{H}} U^{n}\right|_{\mathcal{H}} \quad \text { for } n \in \mathbb{N} \text {, }
$$

Copyright 2016 by the Tusi Mathematical Research Group.

Received Apr. 16, 2015; Accepted Nov. 19, 2015.

${ }^{*}$ Corresponding author.

2010 Mathematics Subject Classification. Primary 47B20; Secondary 47B40, 47A20, 47B37.

Keywords. isometries, Szegö measures, Wold decomposition, wandering vectors, invariant subspaces. 


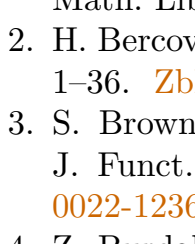

Banach J. Math. Anal. 10 (2016), no. 3, 593-607

http://dx.doi.org/10.1215/17358787-3607420

ISSN: $1735-8787$ (electronic)

http://projecteuclid.org/bjma

ANALYSIS

\title{
SZEGÖ-TYPE DECOMPOSITIONS FOR ISOMETRIES
}

\author{
ZBIGNIEW BURDAK, ${ }^{1}$ MAREK KOSIEK, ${ }^{2}$ \\ PATRYK PAGACZ, $2^{*}$ and MAREK SŁOCIŃSKI ${ }^{2}$ \\ Communicated by F. Kittaneh
}

\begin{abstract}
The notion of Szegö-type properties of positive Borel measures is well known and widely exploited. In this paper, we consider a class of orthogonal decompositions of isometries on Hilbert spaces which correspond to Szegö-type properties of their elementary measures. Our decompositions are closely connected with some special families of invariant subspaces. It is shown that this connection holds for the decomposition constructed in the paper. We illustrate our results with several examples. We also give a short proof of Mlak's theorem on the elementary measures of completely nonunitary contractions.
\end{abstract}

\section{INTRODUCTION AND PRELIMINARIES}

Let $\mathcal{B}(\mathcal{H})$ denote the algebra of all bounded linear operators on a complex Hilbert space $\mathcal{H}$. For a given isometry $V \in \mathcal{B}(\mathcal{H})$, denote by $\mathcal{H}=\mathcal{H}_{u} \oplus \mathcal{H}_{s}$ its Wold decomposition and by $E$ the spectral measure of its minimal unitary extension. For every $x \in \mathcal{H}$ the mapping $\mu_{x}: \mathbb{B}(\mathbb{T}) \ni \omega \mapsto\langle E(\omega) x, x\rangle$ is a positive Borel measure, where $\mathbb{B}(\mathbb{T})$ denotes the $\sigma$-algebra of all Borel subsets of the unit circle $\mathbb{T}$. The measure $\mu_{x}$ is called the elementary measure of $x$ (and $V$ ).

Recall that a unitary operator $U \in \mathcal{B}(\mathcal{K})$ is called a unitary dilation of a contraction $T \in \mathcal{B}(\mathcal{H})$ if

$$
T^{n}=\left.P_{\mathcal{H}} U^{n}\right|_{\mathcal{H}} \quad \text { for } n \in \mathbb{N} \text {, }
$$

Copyright 2016 by the Tusi Mathematical Research Group.

Received Apr. 16, 2015; Accepted Nov. 19, 2015.

${ }^{*}$ Corresponding author.

2010 Mathematics Subject Classification. Primary 47B20; Secondary 47B40, 47A20, 47B37.

Keywords. isometries, Szegö measures, Wold decomposition, wandering vectors, invariant subspaces. 